\title{
Guiding Appropriate Timing of Laser Irradiation by Polymeric Micelles for Maximizing Chemo-Photodynamic Therapy
}

This article was published in the following Dove Press journal: International Journal of Nanomedicine

\author{
Yun Zhu ${ }^{1,2, *}$ \\ Fangying $\mathrm{Yu}^{1, *}$ \\ Yanan Tan ${ }^{1,3}$ \\ Lijuan Wen ${ }^{1,4}$ \\ Yinghong $\mathrm{Li}^{5}$ \\ Hong Yuan (D) \\ Fuqiang $\mathrm{Hu}^{\prime}$ \\ 'College of Pharmaceutical Science, \\ Zhejiang University, Hangzhou, People's \\ Republic of China; ${ }^{2}$ Nanjing Drum Tower \\ Hospital, The Affiliated Hospital of \\ Nanjing University Medical School, \\ Nanjing, People's Republic of China; \\ ${ }^{3}$ Department of Clinical Oncology, The \\ University of Hong Kong-Shenzhen \\ Hospital, Shenzhen, People's Republic of \\ China; ${ }^{4}$ National Engineering Research \\ Center for Modernization of Traditional \\ Chinese Medicine-Hakka Medical \\ Resources Branch, College of Pharmacy, \\ Gannan Medical University, Ganzhou, \\ People's Republic of China; ${ }^{5}$ Zhejiang \\ Institute for Food and Drug Control, \\ Hangzhou, People's Republic of China
}

*These authors contributed equally to this work
Background: Photoactivity "on-off" switchable nano-agents could shield phototoxicity until reaching target region, which immensely promoted photodynamic therapy. However, the masking ratio of nano-agents in vivo was dynamic and positively correlated with the phototoxicity induced by laser irradiation, in which case the timing of laser irradiation was unpredictable to maximize antitumor efficacy.

Methods: Herein, low molecular weight chitosan and hydrophobic polymethylacrylamide derivatives were linked via GSH cleavable 3, 3'-dithiodipropionic acid to construct polymeric micelles (Ce6-CSPD). The doxorubicin loading nano-agent (Ce6-CSPD/DOX) could quench both photoactivity and fluorescence of photosensitizer chlorin e6 (Ce6) and doxorubicin (DOX) under physiological condition by homo-fluorescence resonance energy transfer (homoFRET).

Results: Once internalized by tumor cells, the photoactivity as well as fluorescence of Ce6 was recovered rapidly when motivated by intracellular high GSH. Specifically, the fluorescence intensity and photoactivity of Ce6 were proven to be positive linear correlated, upon which appropriate timing of laser irradiation could be determined by referring to the dynamic fluorescence intensity in vivo. In addition, the theranostic nano-agents also possessed the capacity of monitoring the DOX release process. Accordingly, under the guidance of fluorescence intensity, the experimental group subjected to laser irradiation at $18 \mathrm{~h}$ postadministration acquired the highest antitumor inhibition efficacy compared to that at four hours and $48 \mathrm{~h}$, which held great potential for maximizing chemophotodynamic therapy and avoiding nonspecific phototoxicity precisely to normal organs.

Conclusion: In summary, we prepared homoFRET-based theranostic nano-agent (Ce6CSPD/DOX) for monitoring PDT precisely and decreasing phototoxicity to normal organs before reaching target region. Under the guidance of dynamic fluorescence intensity, the appropriate laser irradiation timing could be monitored to maximize antitumor therapy efficacy, which offered opportunities for monitoring efficiency of chemo-photodynamic therapy in a timely and accurate manner.

Keywords: theranostic, photodynamic therapy, tumor, micelle

\section{Introduction}

Cancers are still leadto millions of human deaths worldwide, although continuous efforts are afforded by scholars. ${ }^{1,2}$ For the moment, chemotherapy and radiotherapy are still the standard strategies for the treatment of tumors. However, inefficiencies and severe side effects generally lead to compromised patient compliance. ${ }^{3-5}$ Thus, novel advancements such as photodynamic therapy (PDT) have gradually become regarded as the clinical candidate for tumor treatment, with advantages of destroying localized tumor regions, minimizing damage of surrounding tissue with high spatiotemporal precision. ${ }^{6,7}$ 
PDT inhibited tumor proliferation and metastasis by generating cytotoxic reactive oxygen species (ROS) with nearinfrared laser irradiation. ${ }^{8,9}$ However, unavoidable light such as sunlight could induce photoactivity during blood circulation. Thus, patients administrated with photodynamic reagent needed to stay in a dark room for several days to avoid irreversible damage, which severely restricts further application in clinics. ${ }^{10-12}$ Accordingly, the photoactivity "on-off" switchable nano-agents could quench photoactivity of photodynamic reagent before reaching the desired locations, which poses great potential in cancer therapy. ${ }^{13-16}$ For instance, Liu et al, utilized MOF nanoparticles as quenchers for photosensitizers, as the MOF structures could be effectively degraded in the presence of intracellular reducing agents, the photoactivity of the photosensitizers would recover after the degradation. ${ }^{17}$ Fan et al, reported that a $\mathrm{MnO}_{2}$ nanosheets where Ce6 were physically absorbed, so that the phototoxicity and optical properties of $\mathrm{Ce} 6$ remained quenched until the nanosheet was destroyed by intracellular redox condition. ${ }^{18}$

Notably, the degree of integrated nano-agents could change owing to the interaction between physicochemical property of materials and physiological/pathological condition in vivo. ${ }^{19-21}$ Thus the shielding ratio was dynamic in vivo, which had significant effect on phototoxicity due to the unpredictable timing of laser irradiation. Most important of all, the degree of depolymerization of micelles directly affected the photodynamic activity. Thus, the appropriate timing of laser irradiation would be very challenging to monitor, strategy for guiding accurate timing of laser irradiation was urgently needed to maximize antitumor efficacy of PDT.

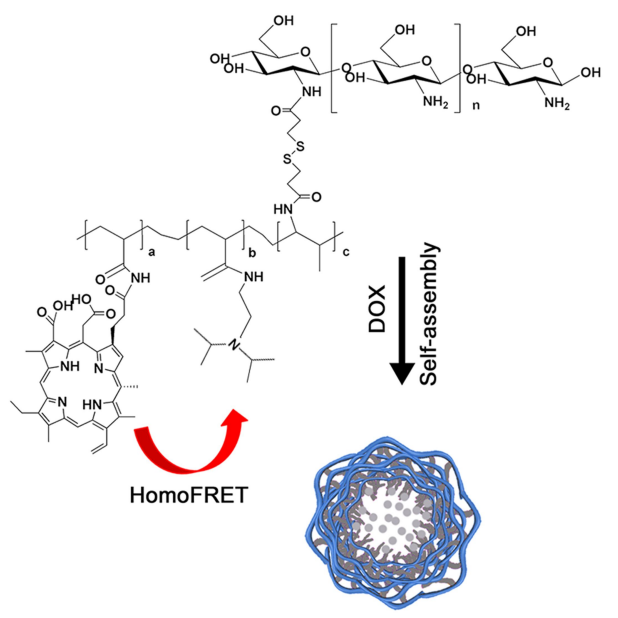

Fluorescence/PDT "off”
To this end, polymeric micelles (Ce6-CSPD) were synthesized to monitor appropriate timing for PDT based on the fluorescence intensity as shown in Scheme 1. Generally, biocompatible chitosan ( $\mathrm{Mw}=7 \mathrm{kDa}$ ) and hydrophobic polymethylacrylamide derivatives, composed of Ce6 (chlorin e6) and N, N-diisopropylethylenediamine, were linked via GSH cleavable 3, 3'-dithiodipropionic acid. Firstly, the doxorubicin (DOX) loading nano-agent (Ce6-CSPD/DOX) could quench PDT photoactivity and fluorescence of $\mathrm{Ce} 6$ under physiological environment. To avoid phototoxicity to normal organs during blood circulation under sunlight, the quenched photodynamic reagent should be guaranteed before reaching desired targets. As noted, the negatively charged surface of Ce6-CSPD /DOX maintained colloidal stability by resisting adsorption of plasma proteins. When the delivered integrated Ce6-CSPD /DOX internalized by tumor cells and motivated by specific high level of redox condition, the photoactivity and fluorescence of $\mathrm{Ce} 6$ could be recovered. The drug release process could be also monitored by virtue of florescence of DOX. Besides, the inner correlation of fluorescence and PDT photoactivity was also explored carefully, which offered opportunity for monitoring laser irradiation timing to maximize chemophotodynamic therapy in vivo.

\section{Materials and Methods \\ Materials}

Ninety-five percent deacetylated chitosan (CSO, Mw = $450 \mathrm{kDa}$, Yuhuan, China) was degraded with enzymes to acquire low molecular weight $\mathrm{CSO}(\mathrm{Mw}=7 \mathrm{kDa})$. 4-dimethylaminopyridine (DMAP), 1-hydroxy-5-pyrrolidinedione (NHS) and 1-ethyl-3-(3-dimethyl-aminopropyl)

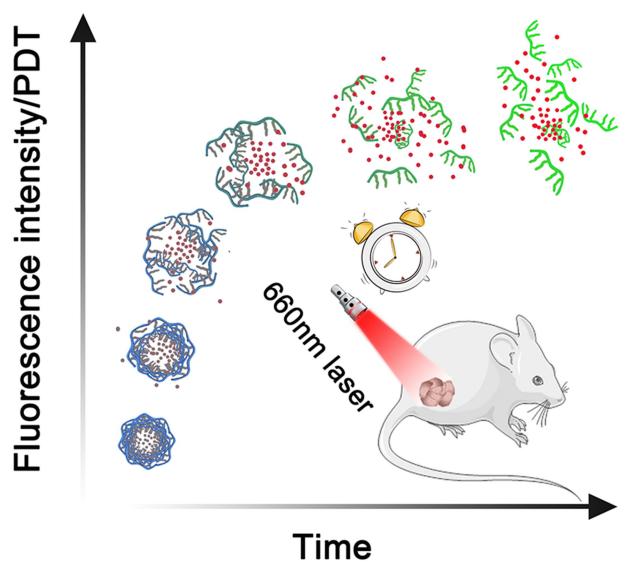


carbodiimide (EDC) were purchased from Shanghai Medpep Co, Ltd (Shanghai, China). Doxorubicin hydrochlorate (DOX.HCl) was obtained from Dalian Meilun Biotech Co., Ltd (Dalian, China). Azodiisobutyronitrile (AIBN), 3-(4, 5-dimethylthiazol-2-yl)-2, 5-diphenyltetrazolium bromide (MTT) were acquired from SigmaAldrich Co. Inc. (St Louis, MO, USA). Methacrylic acid, methacrylamide, N, N-diisopropylethylenediamine were purchased from Aladdin Reagent Database Inc. (Shanghai, China). L-Glutathione (GSH) were purchased from Sigma-Aldrich. The 3, 3'-dithiodipropionic acid (DTPA) and indocyanine green (ICG) were purchased from Tokyo Chemical Industry (Tokyo, Japan). DMEM and trypsin were purchased from Gibco-BRL Life Technologies (Thermo Fisher Scientific, Waltham, MA, USA). Fetal calf serum was purchased from Sijiqing Biology Engineering Materials Co., Ltd (Zhejiang, China). Other chemicals used were of chromatographic grade or analytical grade.

\section{Cells}

4T1 breast tumor cells and human breast cancer cell line MCF-7 were purchased from the Cell Bank of Shanghai Institute of Biochemistry and Cell Biology, Chinese Academy of Sciences (Shanghai, China) and cultured in DMEM supplemented with $10 \%$ fetal calf serum $(\mathrm{v} / \mathrm{v})$, $10000 \mathrm{U} / \mathrm{mL}^{-1}$ streptomycin and $10000 \mathrm{U} / \mathrm{mL}^{-1}$ penicillin at $37^{\circ} \mathrm{C}$ in a humidified incubator with $5 \% \mathrm{CO}_{2}$.

\section{Synthesis and Characterization of the Ce6-CSPD}

Briefly, methacrylic acid and N, N-diisopropylethylenediamine was firstly added into dichloromethane to gain the monomer for polymerization reaction at the feeding molar ratio of 1.2:1 (Figure 1). $\mathrm{H}_{2} \mathrm{O}$ was added to extract water soluble monomer for three times. Then, the collected monomer passed through an $\mathrm{Al}_{2} \mathrm{O}_{3}$ column three times to remove the polymerization inhibitor and catalyst. Finally, the water in the mixture was removed by rotary evaporator as

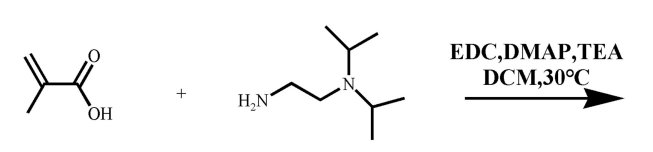

Methacrylic acid N,N-diisopropylethylenediamine
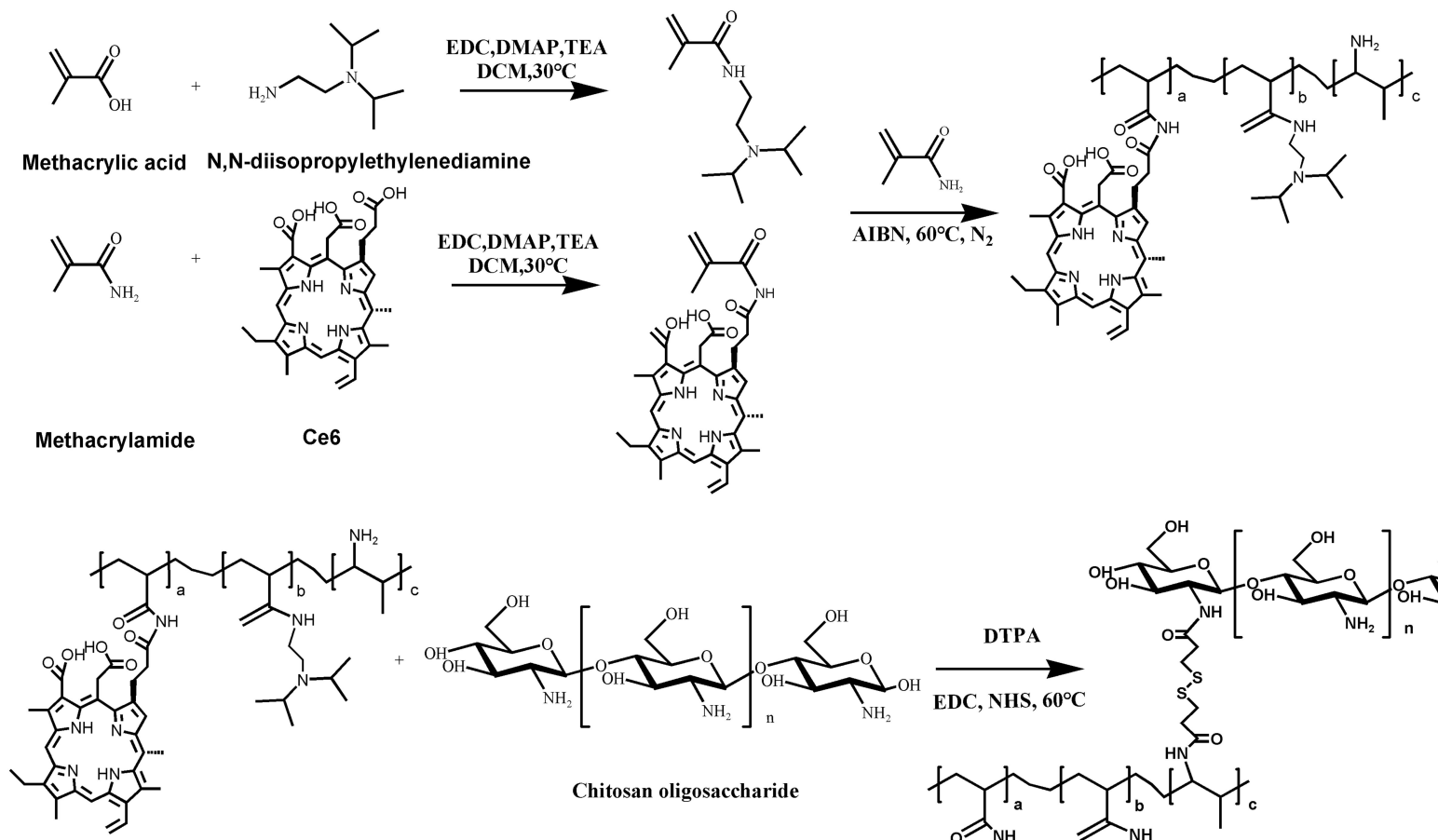

Chitosan oligosaccharide

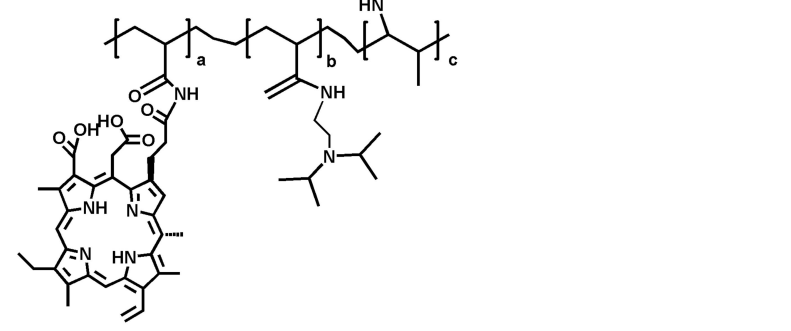

Figure I Illustration of synthetic route of polymeric Ce6-CSPD. 
reported before. ${ }^{22}$ Methacrylamide (mAm) could react with $\mathrm{Ce} 6$ under $\mathrm{N}_{2}$ to get $\mathrm{Ce} 6$ derived monomer and purified as the procedure above. To acquire the hydrophobic co-polymerized fragments, $100 \mathrm{mg}$ Ce6 derived monomer, $1 \mathrm{~g} \mathrm{~N}$, $\mathrm{N}$-diisopropylethylenediamine derived monomer, $60 \mathrm{mg}$ $\mathrm{mAm}$ and $16 \mathrm{mg}$ AIBN were dissolved into DMSO. The oxygen was kept off by pumping nitrogen for $0.5 \mathrm{~h}$. The reaction mixture was subsequently polymerized in $60^{\circ} \mathrm{C}$ water bath kettle for six hours under nitrogen protection. The Ce6 derived monomer based polymer (Ce6-PDPA) were precipitated in a 10-fold excess volume of ethyl acetate for three times. The precipitate was then collected and stored at $-20^{\circ} \mathrm{C}$.

The Ce6-CSPD was synthesized through the amidation reaction between chitosan $(\mathrm{Mw}=7 \quad \mathrm{kDa}), 3, \quad 3^{\prime}-$ dithiodipropionic acid (DTPA) and polymerized Ce6-PDPA. Briefly, $16 \mathrm{mg}$ DTPA, $58 \mathrm{mg}$ EDC, $30 \mathrm{mg}$ NHS and appropriated $\mathrm{HCl}$ were added into a flask and stirred in $2.0 \mathrm{~mL}$ DMF at $60^{\circ} \mathrm{C}$ for two hours. One hundred milligrams polymerized monomer were also dissolved in $1 \mathrm{~mL}$ DMSO and stirred at $60^{\circ} \mathrm{C}$ for two hours. Then the two solutions were mixed for further reaction at $60^{\circ} \mathrm{C}$ for three days. Then, $58 \mathrm{mg}$ EDC, $30 \mathrm{mg}$ NHS dissolved in $3 \mathrm{~mL}$ DMF were placed into the reaction mixture at $60^{\circ} \mathrm{C}$ for two hours, $12 \mathrm{mg} \mathrm{CSO}(\mathrm{Mw}=7$ $\mathrm{kDa}$ ) dissolved in $1 \mathrm{~mL}$ water was placed into the reaction mixture above for the further synthesis process at $60^{\circ} \mathrm{C}$ for another three days. Subsequently, the mixture was dialyzed (MWCO $=14 \mathrm{kDa}$ ) against deionized water for $48 \mathrm{~h}$ and the $\mathrm{pH}$ was adjusted to 7.4 with dilute $\mathrm{HCl}$. Afterwards, the products were then lyophilized and stored at $-20^{\circ} \mathrm{C}$.

Molecular weight of the Ce6-PDPA was determined using gel permeation chromatography (GPC) with DMSO as a mobile phase by PLgel MIXED-C columns (particle size: $5 \mu \mathrm{m}$; dimensions: $7.5 \mathrm{~mm} \times 300 \mathrm{~mm}$ ). ${ }^{23}$ The ${ }^{1} \mathrm{H}$ NMR spectra of the products were confirmed by an NMR spectrometer (AC-80, BrukerBioSpin, karlsruh, Germany). DTPA, Ce6-PDPA were dissolved in DMSO- $_{6}$ at concentrations of $10 \mathrm{mg} / \mathrm{mL}^{-1}$. Chitosan and CSO-SS-PDPA were dissolved in $\mathrm{D}_{2} \mathrm{O}$ at the same concentration. The ratio of conjugated $\mathrm{Ce} 6$ was measured by the fluorescence spectrophotometer.

\section{Evaluation of the Characterization of Ce6-CSPD/DOX}

DOX base loaded Ce6-CSPD (Ce6-CSPD/DOX) was obtained through the hydrophobic interaction as described with some modification. ${ }^{24}$ Briefly, $2 \mathrm{mg} / \mathrm{mL}^{-1}$ of Ce6-CSPD solution was prepared, the DOX dissolved in DMSO at the concentration of $2 \mathrm{mg} / \mathrm{mL}^{-1}$ was added dropwise in weight of $10 \%(\mathrm{w} / \mathrm{w})$ with continuous stirring for $10 \mathrm{~min}$. The mixed solution was dialyzed against water for $24 \mathrm{~h}(\mathrm{MWCO}=14$ $\mathrm{kDa}$ ). The dialyzed solution was centrifuged at $5000 \mathrm{rpm} /$ $\min ^{-1}$ for 15 min to remove unloaded DOX. The hydrodynamic diameters of the Ce6-CSPD and Ce6-CSPD/DOX were measured by the Zetasizer. To evaluate drugs encapsulation capacity of Ce6-CSPD/DOX, $1.98 \mathrm{~mL}$ DMSO was added to $0.02 \mathrm{~mL}$ Ce6-CSPD/DOX to extract the encapsulated DOX. The DOX concentration was calculated according to the standard curve of DOX measured by fluorescence spectrophotometer. The drug encapsulation efficiency (EE $\%$ ) and drug loading efficiency (DL \%) of Ce6-CSPD/DOX were calculated as following:

$$
\begin{aligned}
& \mathrm{EE}(\%)=\frac{\text { weight of DOX in micelles }}{\text { weight of DOX in feed }} \times 100 \% \\
& \mathrm{DL}(\%) \frac{\text { weight of DOX in micelles }}{\text { weight of DOX and polymer }} \times 100 \%
\end{aligned}
$$

The clear morphological of Ce6-CSPD and Ce6-CSPD /DOX was visualized by scanning electron microscope (SEM, GEMINI SEM 300, Oberkochen, Germany). The FTIR spectra for DOX, Ce6 and Ce6-CSPD/DOX were taken over the range from 4000 to $500 \mathrm{~cm}^{-1}$ by virtue of a FTIR spectrometer (Jasco II-4100, Kyot, Japan) to verify the successful preparation of Ce6-CSPD/DOX. The dialysis membrane method was adopted to monitor redoxresponsive drug release profile of DOX loaded micelles at varied GSH concentrations.

\section{Colloidal Stability of Ce6-CSPD/DOX in vitro and Hemolysis Studies}

The positive charged polyplexes was prepared by PEI and protamine at a ratio of $1: 1$, which was set as control group. ${ }^{22}$ Both the polyplexes and Ce6-CSPD/DOX were also incubated with $3 \%$ FBS solution ( $\mathrm{pH}$ 7.4) for different times. The alteration in turbidity at $350 \mathrm{~nm}$ was monitored with an UV-visible light spectrophotometer. ${ }^{25}$ In addition, the morphological of control polyplexes and Ce6-CSPD /DOX before and after 3\% FBS incubation was visualized by transmission electron microscope (TEM, JEOL JEM1230, Tokyo, Japan).

For the hemolysis assay, red blood cells (RBCs) were first obtained from a New Zealand rabbit, which was later diluted to $2 \%$ suspension with saline solution. The Ce6CSPD were added into $2 \%$ RBCs suspension at the ratio of $1: 1$, achieving the designated concentrations $(0.025,0.05$, 
$0.1,0.2,0.4,0.8 \mathrm{mg} / \mathrm{mL}$ ) and incubated at $37^{\circ} \mathrm{C}$ for one hour. Saline and distilled water were adopted as the as negative ( $0 \%$ hemolysis) and positive controls $(100 \%$ hemolysis) respectively under the same condition.

\section{Fluorescence Characterization of Ce6-CSPD/DOX in vitro}

The fluorescence characterization of Ce6-CSPD/DOX was evaluated to confirm the colloidal stability and responsiveness in vitro. Briefly, $1.0 \mathrm{mg} / \mathrm{mL}^{-1}$ (concentration of Ce6CSPD) Ce6-CSPD/DOX was dispersed into $\mathrm{pH}$ 7.4 PBS (containing 3\% FBS, w/v). The fluorescence intensity of Ce6 and DOX incubated without/with $10 \mathrm{mM} \mathrm{GSH}$ for different time was then evaluated by fluorescence spectrophotometer. Meanwhile, the images of Ce6-CSPD/DOX were also visualized by a Maestro in vivo imaging system (CRI Inc., Woburn, MA, USA).

Subsequently, the inner correlation between fluorescence intensity of Ce6 and produced ROS under $660 \mathrm{~nm}$ laser irradiation (two minutes, $200 \mathrm{~mW} / \mathrm{cm}^{2}$ ) were further investigated. Briefly, $5 \times 10^{4} 4 \mathrm{~T} 1$ and MCF-7 cells were seeded into 24 -well plates and incubated at $37^{\circ} \mathrm{C}$ for 12 $\mathrm{h}$, respectively. Appropriate amounts of Ce6-CSPD/DOX were added into each well and incubated for different times. Tumor cells were further irradiated under 200 $\mathrm{mW} / \mathrm{cm}^{2}$ laser for two minutes. Afterwards, the probe staining levels of ROS (DCFH-DA) was incubated for 30 $\min$ at $37^{\circ} \mathrm{C}$. The supernate was discarded and cells were washed with PBS three times. Then cells were harvested with trypsin solution and suspended into $1 \mathrm{~mL}$ PBS. Finally, the collected cells were immediately analyzed with fluorescence spectrophotometer for the fluorescence intensity of DCFH-DA and Ce6.

To monitor the appropriate timing of NIR-laser irradiation, $5 \times 10^{4} 4 \mathrm{~T} 1$ and MCF-7 cells were also plated into 24well plates and incubated at $37^{\circ} \mathrm{C}$ overnight, respectively. Then appropriate amounts of coumarin 6 loaded micelles (Ce6-CSPD/C6) was added and incubated for appointed times. The supernate was discarded and cells were washed with PBS three times. Afterwards, the cells were digested with tyrisin and dispersed into $1 \mathrm{~mL}$ PBS, then the fluorescence the fluorescence intensity of C6 and Ce6 were measured for track of amounts and the integrity of internalized micelles.

The fluorescence intensity changing patterns of Ce6CSPD/DOX was also investigated in 4T1 cells by CLSM. Briefly, $2 \times 10^{4} \mathrm{~mL}^{-1} 4 \mathrm{~T} 1$ cells were seeded in culture dish and incubated overnight. Then, the cultured medium was replaced with fresh medium containing Ce6-CSPD/DOX and incubated for different times. Then, the live tumor cells were visualized by CLSM to investigate intracellular behaviors of fluorescence of $\mathrm{Ce} 6$ and DOX. Besides, the well for $24 \mathrm{~h}$ incubation was further added amounts of GSH (final concentration was $10 \mathrm{mM}$ ) for further one minute and visualized by CLSM immediately.

\section{MTT Assays}

After exploration of timing for NIR laser irradiation, the MTT assays were firstly adapted to confirm the applicability of fluorescence guided PDT. The appropriate concentration of polymer was explored. Briefly, 4T1 cells were plated into 96-well plates at the density of $2 \times 10^{3}$ cells/well and incubated at $37^{\circ} \mathrm{C}$ overnight. Gradient concentration of Ce6-CSPD was added into different wells and further incubated for $48 \mathrm{~h}$ without/with NIR laser irradiation (200 $\mathrm{mW} / \mathrm{cm}^{2}$ ) for two minutes. Twenty microliters MTT $\left(5.0 \mathrm{mg} / \mathrm{mL}^{-1}\right)$ was added and further incubated for four hours at $37^{\circ} \mathrm{C}$. Then, the medium was discarded, $100 \mu \mathrm{L}$ DMSO was then added to dissolve the formazan crystals. The absorbance of each well at $570 \mathrm{~nm}$ was measured by a universal microplate reader (EL800, BIO-TEK Instruments Inc., Winooski, USA).

Afterwards, the incubation time dependent cytotoxicity for Ce6-CSPD at the appointed concentration was evaluated. In brief, 4T1 cells were plated into 96-well plates at a density of $2 \times 10^{3}$ cells/well and incubated at $37^{\circ} \mathrm{C}$ overnight; $50 \mu \mathrm{g} / \mathrm{mL}^{-1}$ Ce6-CSPD was added into wells for different incubation times. Then, drug-treated cells were irradiatated with $660 \mathrm{~nm}$ NIR laser irradiation as the parameters before. The detection of cell viability was measured as before.

\section{Fluorescence Characterization and Antitumor Efficacy of Ce6-CSPD/DOX in vivo}

Female Balb/C nude mice (6-8 weeks) were purchased from the Shanghai Silaike Laboratory Animal Limited Liability Company and fed in SPF cleaning room at standard condition. (All the studies were approved by the Zhejiang University Laboratory Animal Welfare Ethics Review Committee and were conducted in accordance with the national regulations and protocols, the ethical approval number for the in vivo experiments was ZJU20170542). After one week's adaption, $1 \times 10^{6}$ cells in $100 \mu \mathrm{L}$ PBS were 
subcutaneously implanted in the flanks of the mice. All the studies were conducted according to the guidelines issued by the Ethical Committee of Zhejiang University. To observe the fluorescence intensity changing patterns of $\mathrm{Ce} 6$ and DOX, Ce6-CSPD/DOX were injected via the tail vein for different times, respectively. Then, tumor biopsies in different groups were excised and visualized by CLSM. In addition, the tumor masses and major organs were excised at 0 $\mathrm{h}$ and $12 \mathrm{~h}$ postinjection, which were observed by the Maestro in vivo imaging system. ${ }^{26}$

When tumor volume reached appropriate $50 \mathrm{~mm}^{3}$, Balb/c mice bearing 4T1 breast cells were randomly divided into six groups and administrated once a day for two days for three times: Control, DOX, Ce6-CSPD/DOX without laser irradiation, Ce6-CSPD/DOX with laser irradiation (660 nm laser irradiation for five minutes with 0.8 $\mathrm{W} / \mathrm{cm}^{2}$ ) at four hours postinjection, Ce6-CSPD/DOX with laser irradiation at $18 \mathrm{~h}$ postinjection, Ce6-CSPD/DOX with laser irradiation at $48 \mathrm{~h}$ postinjection. Each group was injected through the tail vein. The total dosage of DOX and Ce6 was $4.5 \mathrm{mg} / \mathrm{kg}$ and $2.4 \mathrm{mg} / \mathrm{kg}$, respectively. The volume of tumors and weight of mice during drug treatment were monitored. The tumor volumes were calculated as the formula of $\mathrm{W}^{2} \times \mathrm{L} / 2$, where $\mathrm{W}$ represented the short axis and $\mathrm{L}$ represented the long axis. Tumor masses were exercised and weighed on day 14 . H\&E staining was adopted to evaluate the structure of tumors and major organs.

\section{Statistical Analysis}

Results are presented as mean \pm standard derivation. The analysis of difference was examined by two-tailed Student's $t$-test. A $p$ value of $p<0.05$ was considered statistically significant.

\section{Results and Discussion}

The synthesis route of polymeric Ce6-CSPD was shown in Figure 1. The ratio of $\mathrm{Ce} 6$ in Ce6-CSPD was $4.38 \%$ as measured by fluorescence spectrophotometer. The chemical structure of Ce6-CSPD was measured by ${ }^{1} \mathrm{H}$ NMR spectroscopy as shown in Figure S1, peaks of Ce6-CSPD at $1.01 \mathrm{ppm}, 1.33 \mathrm{ppm}, 1.66 \mathrm{ppm}$ and $3.62 \mathrm{ppm}$ could be attributed to proton of $\mathrm{Ce} 6$, peaks at $3.74 \mathrm{ppm}$ could be assigned to proton of chitosan. The peaks at $2.70 \mathrm{ppm}$ and $2.84 \mathrm{ppm}$ were attributed to protons in DTPA. FT-IR spectrum in Figure S2 demonstrated the successful preparation of Ce6-CSPD/DOX. Besides, UV-vis spectrophotometer was utilized to scan the spectrum of $\mathrm{Ce} 6$ and
Ce6-CSPD as shown in Figure S3, which further demonstrated the successful synthesis of Ce6-CSPD.

The polymeric micelles were prepared by self-aggregating in aqueous phase through hydrophobic interaction between Ce6 and introduced N, N-diisopropylethylenediamine. The diameter of Ce6-CSPD was recorded as $341.12 \pm 29.74 \mathrm{~nm}$ by dynamic light scattering (DLS) (Table 1). After loading DOX, the drug- loading micelles (Ce6-CSPD/DOX) presented a hydrodynamic particle size of $96.98 \pm 1.87 \mathrm{~nm}$ with the drug-loading efficiency (DL) of $6.75 \%$ and encapsulation efficiency (EE) of $72.39 \%$. The decreased diameter could be attributed to the enhanced hydrophobic interaction between the guest drugs. The zeta potentials of Ce6-CSPD and Ce6CSPD/DOX were evaluated to be $-30.38 \pm 2.85 \mathrm{mV}$ and -8.18 $\pm 1.18 \mathrm{mV}$, respectively.

To avoid producing unscheduled phototoxicity of photodynamic nano-agents to normal organs before reaching targeted tissues, integrated morphology of Ce6-CSPD/DOX was most essential during blood circulation. However, DDSs with positive surface tent to aggregate as a result of the interaction with negative charged complex protein milieus in vivo, ${ }^{27,28}$ in which case negative/neutral DDSs were recommended. As mentioned above, the zeta potentials of Ce6-CSPD/DOX had been evaluated as negative. In addition, the stability in vitro was further measured as shown in Figure 2. The Ce6-CSPD/DOX was incubated with 3\% (w/v) FBS for $24 \mathrm{~h}$ and the positive charged polyplexes $(2.72 \pm 1.25$ $\mathrm{mV}$ ) was set as the control group. Images of both micelles were also visualized by TEM and SEM. The results in Figure $2 \mathrm{~A}$ and Figure S4 depicted that the morphology of polyplexes changed dramatically and lost the regular spheroid form after incubating with FBS. In contrast, Ce6-CSPD /DOX still maintained uniform dispersibility and original morphology. The images were also captured as shown in Figure 2B, Ce6-CSPD/DOX exhibited transparent condition while the control group turned turbid immediately the FBS was added. $^{22}$

To further confirm the stability of Ce6-CSPD/DOX, the turbidity at $350 \mathrm{~nm}$ of complex were investigated by UV-vis

Table I Characterization of Polymeric Micelles

\begin{tabular}{|l|l|l|l|l|l|}
\hline Micelles & $\begin{array}{l}\text { Size } \\
(\mathbf{n m})\end{array}$ & $\begin{array}{l}\text { Zeta Potentials } \\
(\mathbf{m V})\end{array}$ & PDI & $\begin{array}{l}\text { DL } \\
(\%)\end{array}$ & $\begin{array}{l}\text { EE } \\
\mathbf{( \% )}\end{array}$ \\
\hline Ce6-CSPD & $\begin{array}{l}341.12 \\
\pm 29.74\end{array}$ & $-30.38 \pm 2.85$ & $\begin{array}{l}0.26 \\
\pm 0.01\end{array}$ & - & - \\
\hline $\begin{array}{l}\text { Ce6-CSPD } \\
\text { IDOX }\end{array}$ & $\begin{array}{l}96.98 \\
\pm 1.87\end{array}$ & $-8.18 \pm 1.18$ & $\begin{array}{l}0.22 \\
\pm 0.02\end{array}$ & 6.75 & 72.39 \\
\hline
\end{tabular}




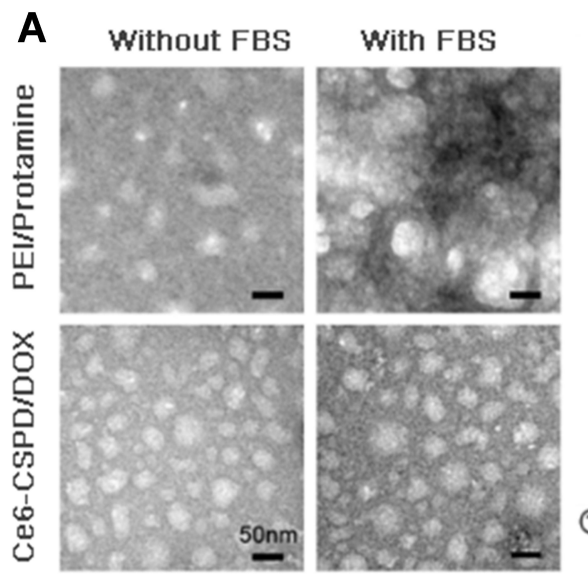

\section{C}

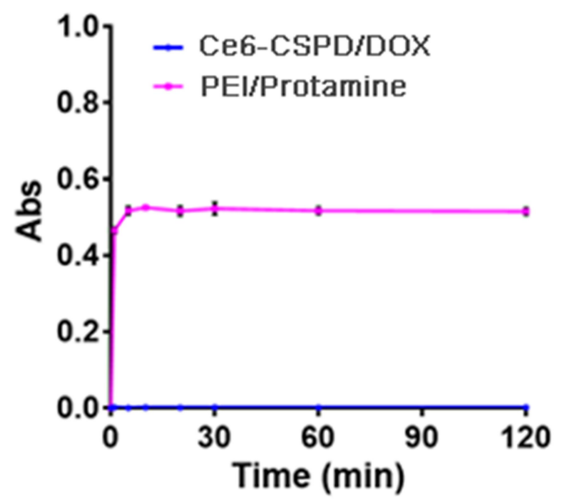

B

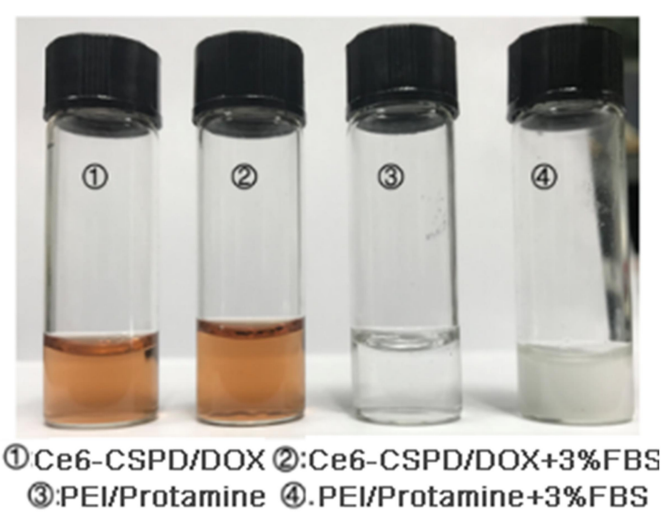

D

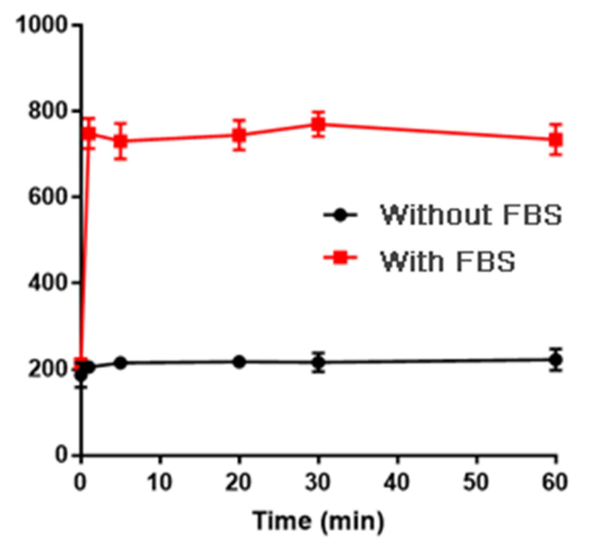

Figure 2 Images of Ce6-CSPD/DOX incubated with 3\% (w/v) FBS and observed by (A) TEM and (B) camera. (C) The absorbance of Ce6-CSPD/DOX and control group at $350 \mathrm{~nm}$ after appointed incubation time with 3\% FBS. (D) Fluorescence intensity of Ce6 after incubating Ce6-CSPD/DOX in pH 7.4 PBS (3\% FBS) without/with I0 mM GSH.

spectrophotometer (Figure 2C). The positive charged polyplexes turned turbid ultrafast and exhibited time-dependent proteins adsorption behavior during the incubation according to the UV absorption data. In contrast, the turbidity of Ce6CSPD/DOX remained unchanged, demonstrating the good stability in vitro. Moreover, the incorporated DOX showed limited effect on the spectrum of Ce6-CSPD/DOX as shown in Figure S5. In summary, the prepared Ce6-CSPD/DOX could keep stable and resist the adsorption of negative charged substances, which could avoid unscheduled disaggregation of micelles, significantly decreasing the phototoxicity of photodynamic nano-agents to normal organs.

Subsequently, the capacity of recovering PDT activity was urgently needed once Ce6-CSPD/DOX delivered to tumor tissues. As reported that the difference of redox potential between the extracellular space and the intracellular space reached $\sim 100-1000$ folds, ${ }^{29}$ in which case the incorporation of GSH cleavable linkers might hold great potentials for micelles' disaggregation and later recovered PDT activity. ${ }^{30-32}$
The fluorescence intensity of Ce6-CSPD/DOX incubated with $3 \%$ (w/v) FBS was investigated by fluorospectro photometer. The fluorescence quenching characterization of Ce6 (Figure 2D) at different times after incubation was observed owing to the HomoFRET effect in the self-assembled state, which also was consistent with results of stability test above. In addition, the fluorescence of encapsulated DOX also showed the same tendency as shown in Figure S6. Upon adding GSH into Ce6-CSPD/DOX, both of Ce6 and DOX showed ultrafast recovery in the fluorescence intensity. Besides, the images of DOX from Ce6-CSPD/DOX was also visualized through maestro in vivo imaging system (CRI Inc.) as shown in Figure S7. The fluorescence intensity of DOX in Ce6-CSPD/DOX without GSH maintained stable and very low, while the fluorescence was rapidly recovered within five minutes after incubation with $10 \mathrm{mM}$ GSH. In vitro DOX releasing profiles of Ce6-CSPD/DOX was also studied as shown in Figure S8, which further confirmed that GSH triggered drug releasing characterization of Ce6-CSPD 
/DOX. Collectively, the results above further demonstrated the good colloidal stability of Ce6-CSPD/DOX under physiological condition and the ultrafast fluorescence recovery capacity of Ce6-CSPD/DOX triggered by specific redox condition.

The correlation of fluorescence intensity and photoactivity was further evaluated. The Ce6-CSPD was added into 24-well plates containing 4T1 or MCF-7 cells for different incubation times and lasered for two minutes at $200 \mathrm{~mW} /$ $\mathrm{cm}^{2}$. The fluorescence spectrophotometer was adopted to measure the fluorescence of $\mathrm{Ce} 6$ and the 2',7'dichlorodihydrofluorescein diacetate (DCFH-DA), ${ }^{29}$ where the DCFH-DA was adopted as a probe indicating the level of reactive oxygen species (ROS). Accordingly, the fluorescence intensity of both probes was matched to evaluate the correlation between the fluorescence intensity and the photoactivity of $\mathrm{Ce} 6$, the results were shown in Figure 3A. The fluorescence intensity and photoactivity of Ce6-CSPD was highly positive correlated, with a $\mathrm{R}^{2}$ value of 0.959 and 0.993 for $4 \mathrm{~T} 1$ and MCF-7 cells respectively, which demonstrated the potential of monitoring appropriate
NIR-laser irradiation timing in virtue of the fluorescence intensity of $\mathrm{Ce} 6$ from integrated $\mathrm{Ce} 6$-CSPD.

Hence, the exact timing for NIR-laser irradiation was subsequently explored. Firstly, the characterization of fluorescence intensity of Ce6-CSPD/DOX triggered by redox condition was monitored in living 4T1 cells by confocal laser scanning microscopy (CLSM) as shown in Figure 3B. It is worth noting that Ce6-CSPD/DOX in the supernate did not show any visible fluorescence due to the quenched state. Visible fluorescence of $\mathrm{Ce} 6$ and DOX appeared after four hours incubation in tumor cells regions. Semiquantitative analysis of Ce6 and DOX was shown in Figure 3C, fluorescence intensity of $\mathrm{Ce} 6$ in Ce6-CSPD/DOX reached the maximum after $10 \mathrm{~h}$ incubation $(p<0.001)$. And the fluorescence intensity at $24 \mathrm{~h}$ was equivalent with that at $10 \mathrm{~h}(p=0.625)$. In addition, the fluorescence intensity of DOX continuously increased during the $24 \mathrm{~h}$.

After $24 \mathrm{~h}$ incubation, we supplemented certain amount of GSH into the supernate (the final concentration was 10 $\mathrm{mM}$ ) for further one minute incubation and visualized the
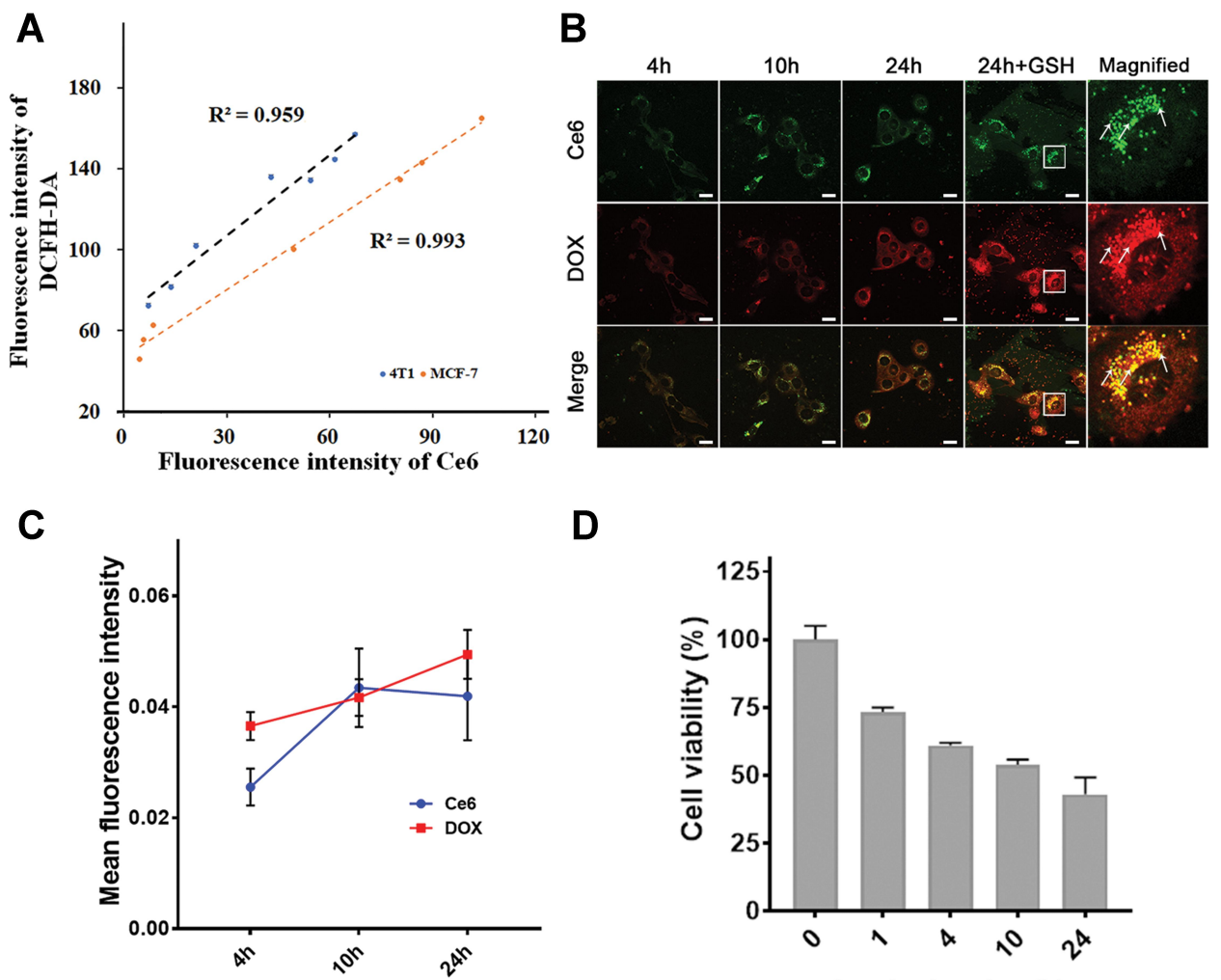

D

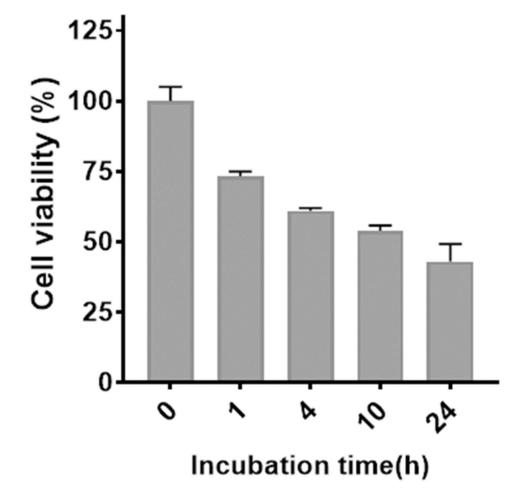

Figure 3 (A) The correlation of fluorescence intensity and produced ROS under $660 \mathrm{~nm}$ laser irradiation for three minutes with $200 \mathrm{~mW} / \mathrm{cm} 2$ on both 4 TI and MCF-7 cells. (B) Dynamic fluorescence intensity and (C) semiquantitative analysis of Ce6 and DOX in live 4TI cells after Ce6-CSPD/DOX were internalized at different incubation time. After $24 \mathrm{~h}$ incubation, adequate GSH (the concentration of GSH in supernate was $10 \mathrm{mM}$ ) was added into medium directly and incubated for one hour at $37^{\circ} \mathrm{C}$. (D) Cytotoxicity evaluation of $50 \mu \mathrm{g} / \mathrm{mL}$ Ce6-CSPD for different incubation time against 4TI cells under $660 \mathrm{~nm}$ laser irradiation (two minutes, $200 \mathrm{~mW} / \mathrm{cm} 2$ ) in vitro. Scare bar: $20 \mu \mathrm{m}$. 
images of relevant fluorescence by CLSM. Both the fluorescence intensity of $\mathrm{Ce} 6$ and DOX increased rapidly and significantly, which could be attributed to the disaggregated micelles in endosome/lysosome (labelled by white arrow) triggered by the added GSH. Besides, amounts of "dot-like" fluorescence was observed in the supernate (fluorescence outside cells) as a result of precipitated hydrophobic fragment from disaggregating Ce6-CSPD/DOX triggered by GSH. The fluorescence changing patterns of Ce6-CSPD /DOX in 4T1 (Figure S9) and MCF-7 (Figure S10) were also investigated by fluorescence spectrophotometer, which also indicated that $10 \mathrm{~h}$ incubation was the appropriate laser irradiation timing for maximizing PDT efficiency.

MTT assays were utilized to further evaluate the feasibility of fluorescence guiding appropriate laser irradiation timing of PDT in vitro. The cytotoxicity of Ce6-CSPD without/with NIR-laser irradiation was evaluated against 4T1 cells as shown in Figure S11. The polymeric material without laser irradiation showed little effect on cell viability, demonstrating good safety of Ce6-CSPD. The Ce6-CSPD also exhibited concentration dependent cytotoxicity under laser irradiation (two minutes, $200 \mathrm{~mW} / \mathrm{cm}^{2}$ ). Then, $660 \mathrm{~nm}$ laser irradiation was performed on groups with different incubation times of Ce6-CSPD $\left(50 \mu \mathrm{g} / \mathrm{mL}^{-1}\right)$ against $4 \mathrm{~T} 1$ cells (Figure 3D), which confirmed that incubation time significantly affects cytotoxicity with laser irradiation. As mentioned above, only the integrity of Ce6-CSPD was destroyed, the PDT efficacy could be recovered, thus, the incubation timedependent cytotoxicity of Ce6-CSPD could be substantially attributed to the increasing concentration of disaggregated Ce6-CSPD (also referred to fluorescence intensity of recovered Ce6), which further confirmed positive correlation between the fluorescence and PDT efficacy of Ce6-CSPD.

Subsequently, the in vivo performances of Ce6-CSPD /DOX were systematically investigated. ICG, as a near infrared fluorescent probe, was labelled on Ce6-CSPD/DOX to monitor the real-time in vivo drug distribution pattern of Ce6CSPD/DOX. As displayed in Figure 4A, the distribution of Ce6-CSPD/DOX in tumors were observed to reach the highest value at a mere two hours postinjection. At each extended time interval, the mice were sacrificed and the main organs as well as tumor tissues were dissected. Ex vivo imaging of ICG in tumor masses and major organs are performed by maestro in vivo imaging system as shown in Figure 4B, which revealed that Ce6-CSPD/DOX holds promising tumortargeting capability compared to other organs.

To investigate whether the fluorescence of Ce6-CSPD /DOX could guide the timing of laser irradiation for maximizing PDT efficacy in vivo, Ce6-CSPD/DOX were administrated through the tail vein and circulated for different times. Afterwards, tumor masses were exercised and observed by CLSM to monitor the fluorescence intensity of Ce6 and DOX as shown in Figure 5A. Only little fluorescence of both $\mathrm{Ce} 6$ and DOX could be visualized at two hours postinjection, and the fluorescence intensity increased over time. The fluorescence intensity of $\mathrm{Ce} 6$ at 12 $\mathrm{h}$ postinjection was as high as that at $24 \mathrm{~h}$ postinjection in vivo. Besides, it was worth noting that only the location of tumor cells showed obvious fluorescence, while the interstitial space showed no fluorescence, which confirmed the specificity of tumor-triggered fluorescence recovery. In addition, the fluorescence changing pattern of DOX in vivo
A

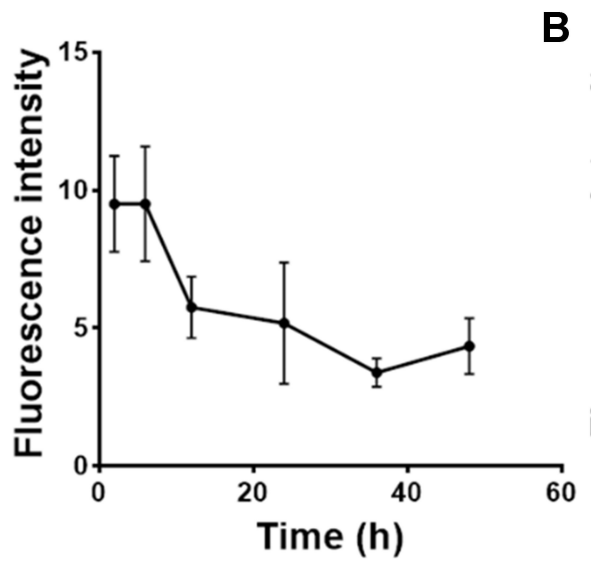

B

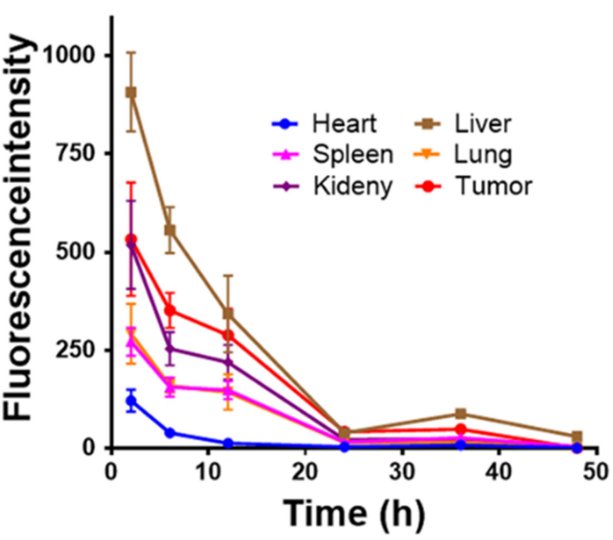

Figure 4 (A) Distribution pattern of Ce6-CSPD/DOX on 4TI breast tumor cells bearing mice. (B) Dynamically changing of Ce6-CSPD/DOX in tumor masses and major organs. 
was almost similar to that of $\mathrm{Ce} 6$, but there were also some differences. Semiquantitative analysis results of $\mathrm{Ce} 6$ and DOX fluorescence were shown in Figure $5 \mathrm{~B}$ and $\mathrm{C}$ respectively. Briefly, the majority of DOX fluorescence was quenched at two hours postinjection of Ce6-CSPD /DOX, while it reached the maximum value at 12 $\mathrm{h}$ postinjection and decreased subsequently. Notably, the time difference between the fluorescence of ICG and Ce6 demonstrated the fact that Ce6-CSPD/DOX could rapidly achieve tumor masses-homing distribution at two hours, while finally the intracellular GSH responsive disaggregation of photodynamic nano-agent was 12 hlate. Thus, exploring the accurate laser irradiation timing for maximizing PDT efficacy was of great significance.

In addition, the fluorescence variation patterns of DOX in major organs was also calculated by the maestro in vivo imaging system as shown in Figure S12. Owing to the disaggregated Ce6-CSPD/DOX triggered by high level of GSH in tumor cells, the fluorescence of DOX in tumor tissues was always the highest during the whole process and increased with prolonged time compared to major organs, further demonstrating the stability of photodynamic nano-agents in major organs and safety in vivo. Meanwhile, the images of DOX fluorescence from Ce6-CSPD/DOX ex vivo at two hours and $12 \mathrm{~h}$ postinjection were shown in Figure S13, the fluorescence intensity was obviously increased in tumor region at $12 \mathrm{~h}$ compared with two hours, which was consistent with the fluorescence section results in Figure 5A.

Subsequently, the antitumor efficacy of Ce6-CSPD /DOX with laser irradiation at different times postinjection was investigated according to the results above. As shown in Figure 6A, DOX and Ce6-CSPD/DOX without laser irradiation only inhibited limited volume of breast tumor when administrated relatively low dosage of DOX (total dosage of DOX was $4.5 \mathrm{mg} / \mathrm{kg}$, which was much less than normally administrated dosage, $p=0.131$ and 0.118 for DOX and Ce6-CSPD/DOX without laser irradiation compared with the control group). ${ }^{23}$ As confirmed above, the degree of micellar disaggregation determined the efficacy of PDT with laser irradiation. Thus, the mice were laser irradiated at different times postinjection (4, 18, and $48 \mathrm{~h}$ ) according to the guidance of fluorescence "on-off" above.

Results exhibited enhanced antitumor efficacy in all groups treated with Ce6-CSPD/DOX under laser irradiation compared to that of DOX group ( $p=0.004$ and 0.042 for laser irradiation at $18 \mathrm{~h}$ compared with that at four hours and $48 \mathrm{~h}$ respectively), which could be attributed to the difference in the resulting cytotoxic ROS level.
A

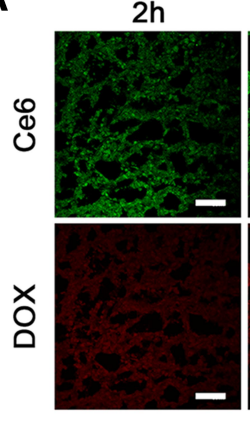

B

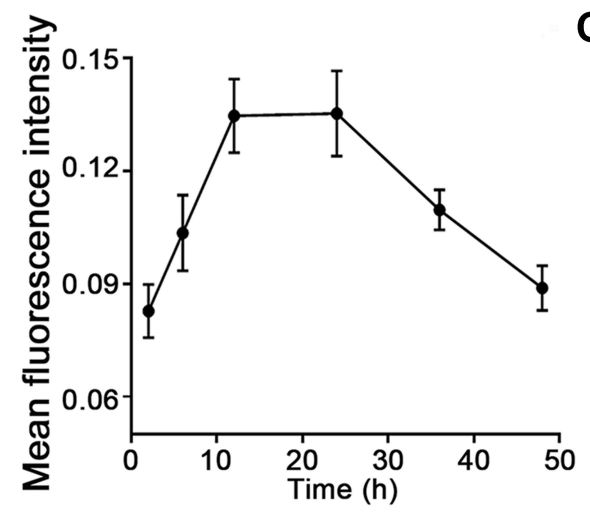

$12 \mathrm{~h}$

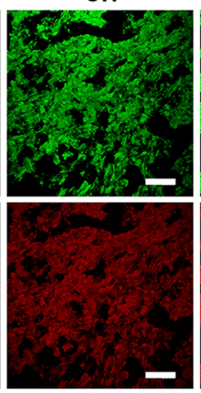

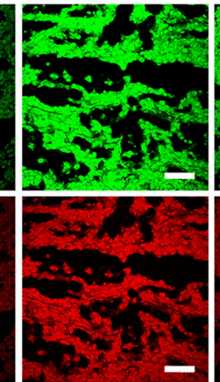

C

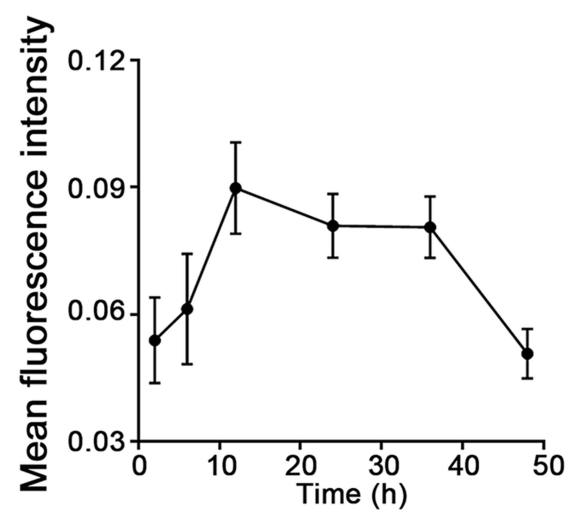

Figure 5 (A) Fluorescence of Ce6 and DOX at 2, 6, 12, 36, and $48 \mathrm{~h}$ postinjection of Ce6-CSPD/DOX visualized by CLSM on tumor bearing Balb/c mice. Semiquantitative analysis of (B) Ce6 and (C) DOX by Imagej software. Scale bar: $50 \mu \mathrm{m}$. 
A

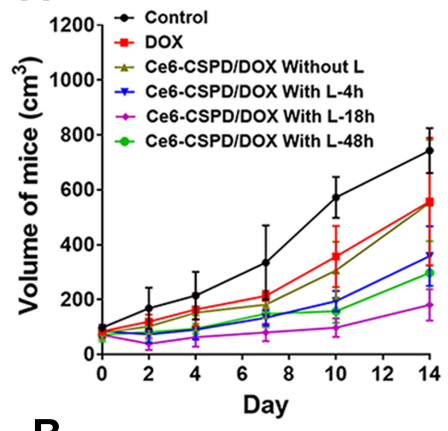

B

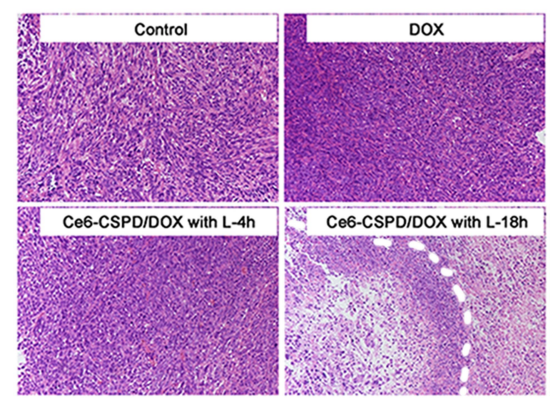

C

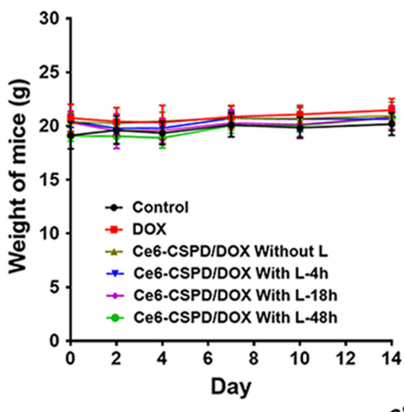

D

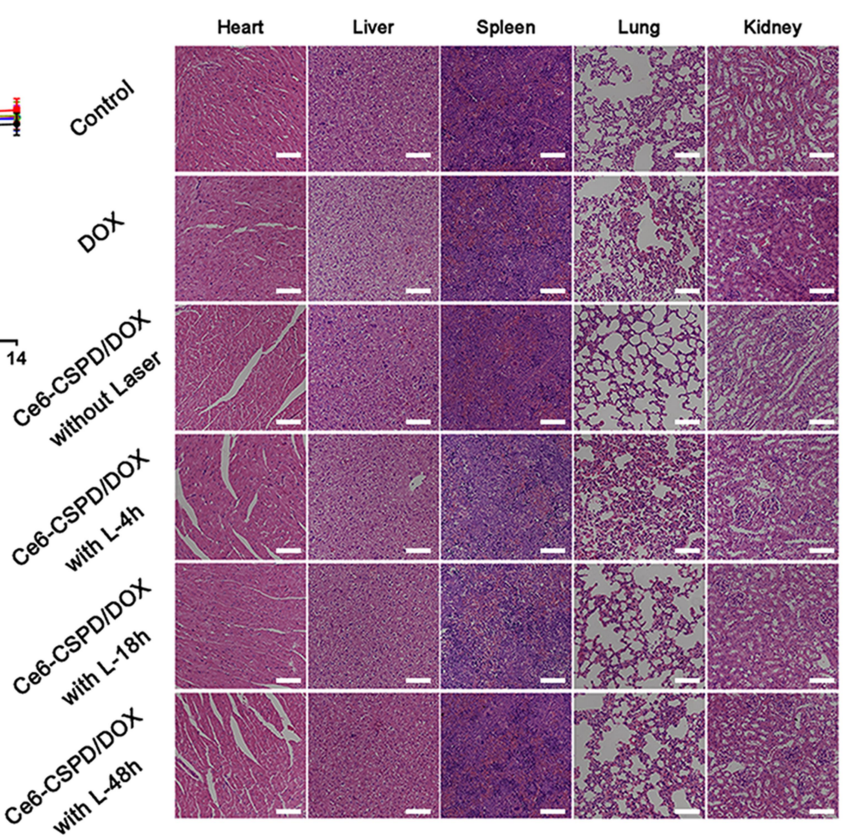

Figure 6 (A) Antitumor efficacy of Ce6-CSPD/DOX at appointed time of laser irradiation. (B) H\&E staining for tumor masses after drugs treatment. The scale bar is 100 $\mu \mathrm{m}$. (C) Weight of mice during drugs treatment. (D) HE staining for major organs after drugs treatment. Scare bar: $100 \mu \mathrm{m}$.

Notably, the timing of laser irradiation after injection significantly affected the capacity of Ce6-CSPD/DOX in vivo tumor inhibition efficacy. Specifically, the mice subjected to laser irradiation at $18 \mathrm{~h}$ postinjection of Ce6-CSPD /DOX showed highest inhibition efficiency compare to other groups. The results of exercised tumor weight in Figure S14 further confirmed the trend of antitumor efficacy.

H\&E staining was adopted to further evaluate the antitumor efficacy after drug treatment as shown in Figure 6B. Amounts of tumor cells underwent severe necrosis after treatment of Ce6-CSPD/DOX with laser irradiation at 18 $\mathrm{h}$ postlaser irradiation, whereas groups subjected to laser irradiation at four hours and $48 \mathrm{~h}$ postadministration exhibited fewer apoptotic regions in the tumor masses due to the quenched PDT photoactivity. In contrast, the antitumor efficacy of Ce6-CSPD/DOX with laser irradiation at four hours postadministration exhibited comparative antitumor efficacy to that of $48 \mathrm{~h}$, which was consistent with results of comparative fluorescence intensity in vivo. The consistence of fluorescence and antitumor efficacy further confirmed the feasibility of maximizing the antitumor therapy efficacy via fluorescence-guiding accurate timing of laser irradiation.
To investigate the safety of Ce6-CSPD/DOX after laser irradiation, weight of tumor bearing mice was monitored as exhibited in Figure 6C, which demonstrated good safety of Ce6-CSPD/DOX in vivo. H\&E staining was also used to stain major organs as shown in Figure 6D. DOX treatment caused serious cardiotoxicities, while no obvious morphologic changes or lesions occurred after NIR laser irradiation, which might be attributed to the PDT quenching effect of Ce6-CSPD/DOX before reaching target region. Hemolysis of Ce6-CSPD after incubation with red blood cells at various concentration for two hours was also performed as shown in Figure S15, which demonstrated good biocompatibility of Ce6-CSPD.

\section{Conclusion}

In summary, we prepared homoFRET based theranostic nano-agent (Ce6-CSPD/DOX) for monitoring PDT precisely and decreasing phototoxicity to normal organs before reaching target region. The photoactivity and fluorescence of photodynamic nano-agent was shielded under physiological condition. The negatively charged Ce6-CSPD/DOX maintained colloidal stability to avoid undesirable phototoxicity by resisting adsorption of proteins during blood circulation. Once internalized by 
tumor cells, the integrated micelles Ce6-CSPD/DOX would disaggregate rapidly due to the specific redox condition in tumor cells, and the activities of fluorescence and photoactivity would restore subsequently. Notably, the fluorescence intensity and photoactivity of Ce6-CSPD/DOX was positive linear correlated. Under the guidance of dynamic fluorescence intensity, the appropriate laser irradiation timing could be monitored to maximize antitumor therapy efficacy, which offered opportunities for monitoring efficiency of chemophotodynamic therapy in a timely and accurate manner.

\section{Abbreviations}

Ce6-CSPD, polymeric micelles; Ce6-CSPD/DOX, doxorubicin loading nano-agent; Ce6, chlorin e6; homoFRET, homofluorescence resonance energy transfer; DOX, doxorubicin; PDT, photodynamic therapy; ROS, reactive oxygen species; CSO, chitosan; NHS, 1-hydroxy-5-pyrrolidinedione; EDC, 1-ethyl-3-(3-dimethyl-aminopropyl) carbodiimide; AIBN, azodiisobutyronitrile; MTT, 3-(4, 5-dimethylthiazol-2-yl)-2, 5-diphenyltetrazolium bromide; GSH, L-glutathione; DTPA, 3, 3'-dithiodipropionic acid; ICG, indocyanine green; mAm, methacrylamide; GPC, gel permeation chromatography; DLS, dynamic light scattering; DL, drug loading efficiency; EE, encapsulation efficiency; TEM, transmission electron microscope; CLSM, confocal laser scanning microscopy.

\section{Funding}

This work was supported by the National Natural Science Foundation of China (81773648) and Zhejiang Provincial Natural Science Foundation of China (D19H30001).

\section{Disclosure}

The authors report no conflicts of interest in this work.

\section{References}

1. Shanmugam V, Selvakumar S, Yeh CS. Near-infrared light-responsive nanomaterials in cancer therapeutics. Chem Soc Rev. 2014;43 (17):6254-6287. doi:10.1039/C4CS00011K

2. Gai SL, Yang GX, Yang PP, et al. Recent advances in functional nanomaterials for light-triggered cancer therapy. Nano Today. 2018;19:146-187. doi:10.1016/j.nantod.2018.02.010

3. Wang T, Wang D, Yu H, et al. Intracellularly acid-switchable multifunctional micelles for combinational photo/chemotherapy of the drug-resistant tumor. ACS Nano. 2016;10(3):3496-3508. doi:10.1021/ acsnano. 5 b07706

4. Lu K, He C, Guo N, et al. Chlorin-based nanoscale metal-organic framework systemically rejects colorectal cancers via synergistic photodynamic therapy and checkpoint blockade immunotherapy. $\mathrm{J}$ Am Chem Soc. 2016;138(38):12502-12510. doi:10.1021/jacs.6b06663
5. Ekladious I, Colson YL, Grinstaff MW. Polymer-drug conjugate therapeutics: advances, insights and prospects. Nat Rev Drug Discov. 2018.

6. Zhang Z, Wang R, Huang X, et al. Self-delivered and self-monitored chemo-photodynamic nanoparticles with light-triggered synergistic antitumor therapies by downregulation of HIF-1alpha and depletion of GSH. ACS Appl Mater Interfaces. 2020; 12(5):5680-94.

7. Xu MS, Yang GX, Bi HT, et al. An intelligent nanoplatform for imaging-guided photodynamic/photothermal/chemo-therapy based on upconversion nanoparticles and $\mathrm{CuS}$ integrated black phosphorus. Chem Eng J. 2020;382.

8. Ethirajan M, Chen YH, Joshi P, Pandey RK. The role of porphyrin chemistry in tumor imaging and photodynamic therapy. Chem Soc Rev. 2011;40(1):340-362. doi:10.1039/B915149B

9. Ding YF, Li S, Liang L, et al. Highly biocompatible chlorin e6-loaded chitosan nanoparticles for improved photodynamic cancer therapy. ACS Appl Mater Interfaces. 2018;10(12):9980-9987. doi:10.1021/acsami.8b01522

10. Idris NM, Gnanasammandhan MK, Zhang J, Ho PC, Mahendran R, Zhang Y. In vivo photodynamic therapy using upconversion nanoparticles as remote-controlled nanotransducers. Nat Med. 2012;18 (10): 1580 . doi: $10.1038 / \mathrm{nm} .2933$

11. Li SY, Liu LH, Rong L, et al. A dual-FRET-based versatile prodrug for real-time drug release monitoring and in situ therapeutic efficacy evaluation. Adv Funct Mater. 2016;25(47):7317-7326. doi:10.1002/ adfm. 201503262

12. Han K, Wang SB, Lei Q, Zhu JY, Zhang XZ. A ratiometric biosensor for aggregation-induced emission-guided precise photodynamic therapy. ACS Nano. 2015;9(10):10268-10277. doi:10.1021/ acsnano. 5 b04243

13. Wang YG, Zhou KJ, Huang G, et al. A nanoparticle-based strategy for the imaging of a broad range of tumours by nonlinear amplification of microenvironment signals. Nat Mater. 2014;13(2):204-212. doi:10.1038/nmat3819

14. Wan YP, Lu GH, Zhang JF, et al. A biocompatible free radical nanogenerator with real-time monitoring capability for high performance sequential hypoxic tumor therapy. Adv Funct Mater. 2019;29 (39): 1903436.

15. Cheng $\mathrm{P}, \mathrm{Pu} \mathrm{K}$. Activatable phototheranostic materials for imaging-guided cancer therapy. ACS Appl Mater Interfaces. 2019;12(5):5286-99.

16. Yu L, Chen Y, Wu M, et al. "Manganese extraction" strategy enables tumor-sensitive biodegradability and theranostics of nanoparticles. J Am Chem Soc. 2016;138(31):9881-9894. doi:10.1021/jacs.6b04299

17. Wang YB, Wu WB, Liu JJ, et al. Cancer-cell-activated photodynamic therapy assisted by cu(II)-based metal-organic framework. ACS Nano. 2019;13(6):6879-6890. doi:10.1021/acsnano.9b01665

18. Fan HH, Yan GB, Zhao ZL, et al. A smart photosensitizer-manganese dioxide nanosystem for enhanced photodynamic therapy by reducing glutathione levels in cancer cells. Angew Chem Int Ed. 2016;55 (18):5477-5482. doi:10.1002/anie.201510748

19. Sahay G, Querbes W, Alabi C, et al. Efficiency of siRNA delivery by lipid nanoparticles is limited by endocytic recycling. Nat Biotechnol. 2013;31(7):653-U119. doi:10.1038/nbt.2614

20. Nel A, Ruoslahti E, Meng H. New insights into "permeability" as in the enhanced permeability and retention effect of cancer nanotherapeutics. ACS Nano. 2017;11(10):9567-9569. doi:10.1021/acsnano.7b07214

21. Kwon YJ. Before and after endosomal escape: roles of stimuli-converting siRNA/polymer interactions in determining gene silencing efficiency. Acc Chem Res. 2012;45(7):1077-1088. doi:10.1021/ar200241v

22. Zhu Y, Meng TL, Tan YN, et al. Negative surface shielded polymeric micelles with colloidal stability for intracellular endosomal/lysosomal escape. Mol Pharm. 2018;15(11):5374-5386. doi:10.1021/acs. molpharmaceut.8b00842 
23. Li W, Huang L, Ying X, et al. Antitumor drug delivery modulated by a polymeric micelle with an upper critical solution temperature. Angew Chem Int Ed Engl. 2015;54(10):3126-3131. doi:10.1002/ anie. 201411524

24. Liu X, Cheng B, Meng T, et al. Synthesis and biological application of BKT-140 peptide modified polymer micelles for treating tumor metastasis with an enhanced cell internalization efficiency. Polym Chem. 2015;7(7):1375-1386. doi:10.1039/C5PY01807B

25. Wang C, Bao X, Ding X, et al. A multifunctional self-dissociative polyethyleneimine derivative coating polymer for enhancing the gene transfection efficiency of DNA/polyethyleneimine polyplexes in vitro and in vivo. Polym Chem. 2015;6(5):780-796. doi:10.1039/ C4PY01135J

26. Hu YW, Du YZ, Liu N, et al. Selective redox-responsive drug release in tumor cells mediated by chitosan based glycolipid-like nanocarrier. J Control Release. 2015;206:91-100. doi:10.1016/j.jconrel.2015.03.018

27. Malcolm DW, Varghese JY, Sorrells JE, Ovitt CE, Benoit DSW. The effects of biological fluids on colloidal stability and siRNA delivery of a pH-responsive micellar nanoparticle delivery system. ACS Nano. 2018;12(1):187-197. doi:10.1021/acsnano.7b05528

28. Fang J, Nakamura H, Maeda H. The EPR effect: unique features of tumor blood vessels for drug delivery, factors involved, and limitations and augmentation of the effect. Adv Drug Deliv Rev. 2011;63 (3):136-151. doi:10.1016/j.addr.2010.04.009
29. Fleige E, Quadir MA, Haag R. Stimuli-responsive polymeric nanocarriers for the controlled transport of active compounds: concepts and applications. Adv Drug Deliv Rev. 2012;64(9):866-884. doi:10.1016/j.addr.2012.01.020

30. Jing $\mathrm{X}$, Zhi $\mathrm{Z}$, Zhang $\mathrm{N}$, et al. Multistage tumor microenvironment-responsive theranostic nanopeanuts: toward multimode imaging guided chemo-photodynamic therapy. Chem Eng J. $2020 ; 385$.

31. Jing $\mathrm{X}$, Zhi $\mathrm{Z}$, Jin $\mathrm{L}$, et al. $\mathrm{pH} /$ redox dual-stimuli-responsive cross-linked polyphosphazene nanoparticles for multimodal imaging-guided chemo-photodynamic therapy. Nanoscale. 2019;11 (19):9457-9467. doi:10.1039/C9NR01194C

32. Liu J, Chen Q, Zhu W, et al. Nanoscale-coordination-polymer-shelled manganese dioxide composite nanoparticles: a multistage redox $/ \mathrm{pH} /$ H2O2-responsive cancer theranostic nanoplatform. Adv Funct Mater. 2017;27(10): 1605926.
International Journal of Nanomedicine

\section{Publish your work in this journal}

The International Journal of Nanomedicine is an international, peerreviewed journal focusing on the application of nanotechnology in diagnostics, therapeutics, and drug delivery systems throughout the biomedical field. This journal is indexed on PubMed Central, MedLine, CAS, SciSearch ${ }^{\circledR}$, Current Contents ${ }^{\circledR} /$ Clinical Medicine,

\section{Dovepress}

Journal Citation Reports/Science Edition, EMBase, Scopus and the Elsevier Bibliographic databases. The manuscript management system is completely online and includes a very quick and fair peer-review system, which is all easy to use. Visit http://www.dovepress.com/ testimonials.php to read real quotes from published authors. 